\title{
MMWAVE MASSIVE ANALOG RELAY MIMO
}

\author{
Kei Sakaguchi' ${ }^{1}$, Takumi Yoneda ${ }^{1}$, Masashi Iwabuchi², Tomoki Murakami² \\ ${ }^{1}$ Tokyo Institute of Technology, 2-12-1 0-okayama, Meguro-ku, Tokyo, Japan. \\ ${ }^{2}$ NTT Access Network Systems Laboratories, 1-1 Hikari-no-oka, Yokosuka-shi, Kanagawa, Japan.
}

NOTE: Corresponding author: Kei Sakaguchi, sakaguchi@mobile.ee.titech.ac.jp

\begin{abstract}
Millimeter-Wave (mmWave) communications are a key technology to realize ultra-high datarate and ultra-low latency wireless communications. Compared with conventional communication systems in the microwave band such as $4 G / L T E$, mmWave communications employ a higher frequency band which allows a wider bandwidth and is suitable for large capacity communications. It is expected to be applied to various use cases such as mmWave cellular networks and vehicular networks. However, due to the strong diffraction loss and the path loss in the mmWave band, it is difficult or even impossible to achieve high channel capacity for User Equipment (UE) located in Non-Line-Of-Sight (NLOS) environments. To solve the problem, the deployment of relay nodes has been considered. In this paper, we consider the use of massive analog Relay Stations (RSs) to relay the transmission signals. By relaying the signals by a large number of RSs, an artificial Multiple-Input Multiple-Output (MIMO) propagation environment can be formed, which enables mmWave MIMO communications to the NLOS environment. We describe a theoretical study of a massive relay MIMO system and extend it to include multi-hop relays. Simulations are conducted, and the numerical results show that the proposed system achieves high data rates even in a grid-like urban environment.
\end{abstract}

Keywords - 5G, mmWave, multi-hop, relay, massive MIMO

\section{INTRODUCTION}

In recent years, the enhanced Mobile Broadband (eMBB) services in the 5th-Generation mobile communication system (5G) are expected to satisfy the increasing traffic demands [1][2]. One of their key enablers is to explore the wireless communication techniques in higher frequency bands, such as the millimeter-Wave (mmWave) band, where the ultra-wide bandwidth enables ultra-high data-rate and ultra-low latency communications [3][4]. MmWave communications are also expected to be applied into and deeply transform a variety of wireless applications such as Vehicle-to-Everything (V2X) communications and Internet of Things (IoT) [5][6].

Though mmWave has relatively high path loss, it can perform long-range communications under a condition of Line-Of-Sight (LOS) [7]. However, the received power of signals in mmWave bands is dramatically reduced by Non-Line-Of-Sight (NLOS) locations or a blockage effect [8]. Since the NLOS probability increases as the communication distance increases, it is difficult to introduce mmWave bands for macro-cells without expanding LOS coverage. Another important issue in mmWave bands is highly spatially correlated channels.
In mmWave bands, due to the propagation loss, the influence of reflected paths and diffracted paths is small and the direct path is dominant. As a result, the number of streams tends to be small in SingleUser (SU) Multiple-Input Multiple-Output (MIMO) [9]. One of the most intuitive and common ways to solve this challenge is to deploy a large number of Base Stations (BSs) as distributed antennas [10]. However, the large-scale deployments of distributed antennas require the high financial cost in both Capital Expenditure (CAPEX) and Operating Expenditure (OPEX). Moreover, it also has to face physical limitations due to the large required installation spaces and power consumption.

Instead of deploying multiple BSs, we focus on distributed relays to solve the above-mentioned issues in mmWave bands. There are several methods for the wireless relay. The Decode-andForward (DF) relay method is one of the typical methods [11], and has been discussed as Integrated Access and Backhaul (IAB) in the standardization of the 3rd Generation Partnership Project (3GPP) Release 16 [12]. In the DF method, since relay nodes perform the decoding and re-encoding operations, noise can be reduced and flexible radio resource control including bandwidth is possible [13]. However, the method has to tolerate the delay due 
to signal processing on relay nodes. Furthermore, since the cost is higher than other relay methods, it is not suitable for deploying a large number of DF nodes. Intelligent Reflecting Surfaces (IRS) is also a remarkable solution. The IRS is a reflector that can switch the reflection direction of radio waves using e.g., meta-surface technologies [14]. However, IRS cannot significantly improve the coverage and capacity since reflection directions are not controlled instantaneously. In this paper, we focus on the Amplifier-and-Forward (AF) relay method with active beam-forming capability. Since the AF method only amplifies signals, delay is almost negligible. Moreover, its cost, size, and power consumption of nodes are relatively small, so that it is suitable to install a large number of AF nodes.

There have been several studies on AF relay methods for mmWave communications [15][16]. The performance of mmWave communications with a full-duplex AF relay was investigated in [15] by considering the effect of beam width and selfinterference. In [16], the basic characteristics of mmWave MIMO communications with multiple AF relays were evaluated under a fixed topology, and it shows potential of the method. When applying the AF relay to mmWave mobile communications, adaptive beam-forming is required to improve communication quality. The conventional AF relay only performs amplification and frequency conversion but adaptive beam-forming has not been considered. Moreover, relay-node selection from massive relay nodes is also important in the case of mobile communications.

In this paper, we propose an mmWave massive analog relay MIMO system for mobile communications by extending the authors' previous work in [17]. A large number of dynamically controllable AF Relay Stations (RSs) are deployed to relay signals for NLOS User Equipment (UE). Adaptive beam-forming in each RS is assumed. A dynamic RS selection algorithm based on UE location is introduced to generate an artificial multipath channel to improve capacity of mmWave MIMO communications. The RSs should have a function of adaptive beam switching for dynamic RS selection and a function of beam tracking to follow the UE's mobility. We start from the formulation in single-hop relay, and then extend it to multi-hop relays to improve mmWave capacity. The full search of optimized route for multi-hop relays requires a huge computational cost for beam selection due to the massive number of RSs. Hence, a fast-routing algorithm is introduced in this paper to maximize channel capacity. Numerical evaluations are conducted to verify the effectiveness of the proposed massive analog relay MIMO system.

The remainder of this paper is organized as follows. In Section 2, a system model of the proposed massive analog relay MIMO is described. In Section 3, expansion to multi-hop relay is explained, and Section 4 describes numerical analysis. Finally, Section 5 concludes and summarizes the paper.

\section{MMWAVE MASSIVE ANALOG RELAY MIMO}

This section describes architecture of the proposed mmWave massive analog relay MIMO that can generate MIMO channels artificially by using a large number of analog RSs.

\subsection{System model of massive analog relay}

Fig. 1 shows the system architecture consisting of one source (BS), one destination (UE), and a massive number of RSs. Because of the compact size, low cost, and low power consumption of analog RSs, it is feasible to massively and non-invasively deploy them in the target environments to enhance or enable mmWave communications.

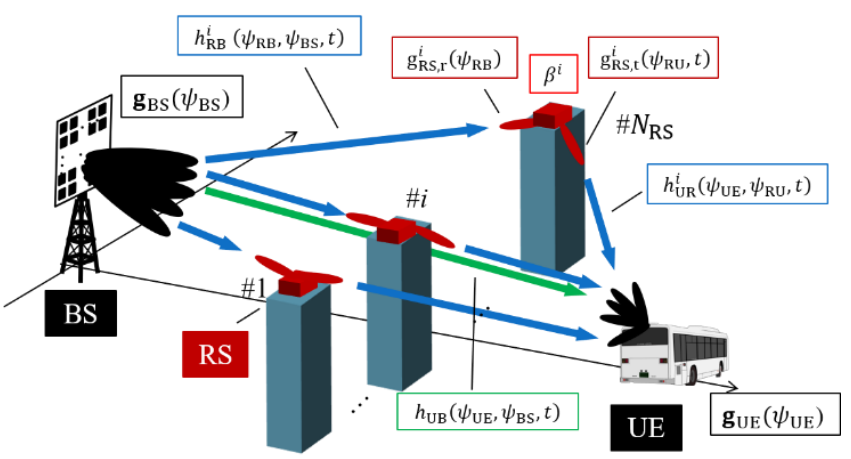

Fig. 1 - System model for mmWave massive analog relay MIMO

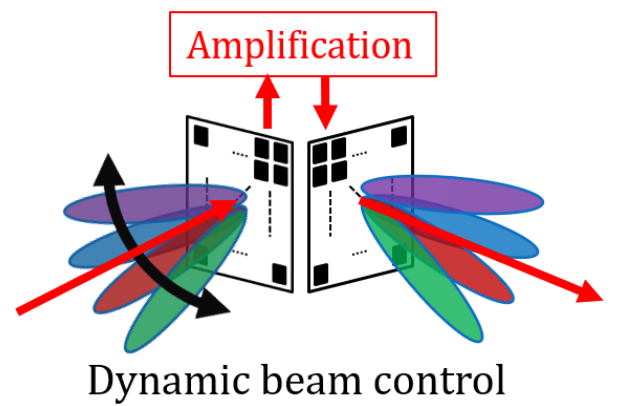

Fig. 2 - mmWave analog RS with beam-forming 
Fig. 2 illustrates the architecture of an analog RS. An analog RS node has two sides, of which one is the receiving side and the other is the transmitting side. As shown in the figure, both sides are assumed to be able to perform high-performance dynamic beam control. The analog RS is supposed to be an AF RS that can amplify the received signal and transmit it without latency.

\subsection{Artificial channel response by massive analog relay}

Artificial propagation channels generated by the massive analog RSs are shown in Fig. 1. The angles of arrival $\psi_{\mathrm{RB}}$ and departure $\psi_{\mathrm{RU}}$ at the RS (BS side and UE side) shown in Fig. 1 are described by the azimuth angle and elevation angle, i.e., $\psi_{\mathrm{RB}}=\left(\theta_{\mathrm{RB}}, \phi_{\mathrm{RB}}\right)$ and $\psi_{\mathrm{RU}}=\left(\theta_{\mathrm{RU}}, \phi_{\mathrm{RU}}\right)$.

The channel response of $\mathrm{BS}^{-\mathrm{RS}_{i}}$ is denoted as $h_{\mathrm{RB}}^{i}\left(\psi_{\mathrm{RB}}, \psi_{\mathrm{BS}}, t\right)$ and that of $\mathrm{RS}_{i}$-UE is denoted as $h_{\mathrm{UR}}^{i}\left(\psi_{\mathrm{UE}}, \psi_{\mathrm{RU}}, t\right) . i=1, \ldots, N_{\mathrm{RS}}$ is $\mathrm{RS}$ index, and $N_{\mathrm{RS}}$ is the number of analog RSs. The channel response of BS-UE (direct path from the BS to the UE) is defined as $h_{\mathrm{UB}}\left(\psi_{\mathrm{UE}}, \psi_{\mathrm{BS}}, t\right)$. The channel response (including the RS antenna directivity) of BS-RS, denoted by $h_{\mathrm{RB}}^{i}\left(\psi_{\mathrm{BS}}, t\right)$, is defined as follows.

$$
\begin{aligned}
& h_{\mathrm{RB}}^{i}\left(\psi_{\mathrm{BS}}, t\right)= \\
& \quad \int_{\psi_{\mathrm{RB}}} g_{\mathrm{RS}, \mathrm{r}}^{i}\left(\psi_{\mathrm{RB}}\right) h_{\mathrm{RB}}^{i}\left(\psi_{\mathrm{RB}}, \psi_{\mathrm{BS}}, t\right) \mathrm{d} \psi_{\mathrm{RB}}
\end{aligned}
$$

where $g_{\mathrm{RS}, \mathrm{r}}^{i}\left(\psi_{\mathrm{RB}}\right)$ is the antenna directivity of $\mathrm{RS}_{i}$ (BS side).

The channel response of $\mathrm{RS}_{i}$-UE, denoted by $h_{\mathrm{UR}}^{i}\left(\psi_{\mathrm{UE}}, t\right)$, is defined as follows.

$$
\begin{aligned}
& h_{\mathrm{UR}}^{i}\left(\psi_{\mathrm{UE}}, t\right)= \\
& \quad \int_{\psi_{\mathrm{RU}}} h_{\mathrm{UR}}^{i}\left(\psi_{\mathrm{UE}}, \psi_{\mathrm{RU}}, t\right) g_{\mathrm{RS}, \mathrm{t}}^{i}\left(\psi_{\mathrm{RU}}, t\right) \mathrm{d} \psi_{\mathrm{RU}}
\end{aligned}
$$

where $g_{\mathrm{RS}, \mathrm{t}}^{i}\left(\psi_{\mathrm{RU}}, t\right)$ is the antenna directivity of $\mathrm{RS}_{i}$ (UE side).

The channel response of $\mathrm{BS}-\mathrm{RS}_{i}$-UE, denoted by $h_{\mathrm{URB}}^{i}\left(\psi_{\mathrm{UE}}, \psi_{\mathrm{BS}}, t\right)$, is defined as follows.

$$
h_{\mathrm{URB}}^{i}\left(\psi_{\mathrm{UE}}, \psi_{\mathrm{BS}}, t\right)=h_{\mathrm{UR}}^{i}\left(\psi_{\mathrm{UE}}, t\right) \beta^{i} h_{\mathrm{RB}}^{i}\left(\psi_{\mathrm{BS}}, t\right)
$$

where $\beta^{i}$ is the amplification coefficient in $\mathrm{RS}_{i}$

The artificial channel response considering all RSs, denoted as $h_{\mathrm{URB}}\left(\psi_{\mathrm{UE}}, \psi_{\mathrm{BS}}, t\right)$, is the summation of all channel responses of BS-UE(considering all RSs) and BS-UE(direct path), as defined as follows.

$$
\begin{aligned}
& h_{\mathrm{URB}}\left(\psi_{\mathrm{UE}}, \psi_{\mathrm{BS}}, t\right)= \\
& \sum_{i=1}^{N_{\mathrm{RS}}} h_{\mathrm{URB}}^{i}\left(\psi_{\mathrm{UE}}, \psi_{\mathrm{BS}}, t\right)+h_{\mathrm{UB}}\left(\psi_{\mathrm{UE}}, \psi_{\mathrm{BS}}, t\right)
\end{aligned}
$$

\subsection{Artificial MIMO channel matrix}

In this paper, the hybrid beam-forming that combines analog BF and digital pre/post-coding is used to efficiently realize massive MIMO transmissions [18]. The BS prepares $N_{\mathrm{BS}}$ antenna beam candidates and stacks them into $N_{\mathrm{BS}} \times 1$ antenna directivity vector $\mathbf{g}_{\mathrm{BS}}$. According to the condition of the artificial channel response in Eq. (4), the BS selects $M$ beams (spatial streams) out of $N_{\mathrm{BS}}$ beam candidates for MIMO multiplexing. On the other hand, the UE always uses $N_{\mathrm{UE}} \times 1$ antenna directivity vector $\mathbf{g}_{\mathrm{UE}}$ stacking $N_{\mathrm{UE}}$ antenna beams.

The $N_{\mathrm{UE}} \times N_{\mathrm{BS}}$ artificial MIMO channel matrix $\mathbf{H}(t)$ including antenna directivities of BS and UE is defined as follows.

$$
\begin{array}{r}
\mathbf{H}(t)=\int_{\psi_{\mathrm{UE}}} \int_{\psi_{\mathrm{BS}}} \mathbf{g}_{\mathrm{UE}}\left(\psi_{\mathrm{UE}}\right) \cdot h_{\mathrm{URB}}\left(\psi_{\mathrm{UE}}, \psi_{\mathrm{BS}}, t\right) \\
\cdot\left(\mathbf{A g}_{\mathrm{BS}}\left(\psi_{\mathrm{BS}}\right)\right)^{\mathrm{T}} \mathrm{d} \psi_{\mathrm{BS}} \mathrm{d} \psi_{\mathrm{UE}}
\end{array}
$$

where $\mathrm{A}$ is $N_{\mathrm{BS}} \times N_{\mathrm{BS}}$ beam selection matrix defined as follows.

$$
\mathbf{A}=\left[\begin{array}{ccc}
\alpha_{1} & & 0 \\
& \ddots & \\
0 & & \alpha_{N_{\mathrm{BS}}}
\end{array}\right]
$$

where $\alpha_{m} \in\{0,1\}, \sum_{m=1}^{N_{\mathrm{BS}}} \alpha_{m}=M . \alpha_{m}$ is 1 if the $m$-th beam is selected, and 0 otherwise. In order to determine A, not only BS beams are selected, but also RSs are used for relaying.

\subsection{Noise vector with massive analog relay}

In the process of relaying signals from the BS to the UE, not only desired signals, but also noise are amplified and forwarded in each RS, which should be taken into consideration in the system analysis. $\mathbf{n}_{\mathrm{RS}}(t)$ is the $N_{\mathrm{UE}} \times 1$ noise signal vector from all $\mathrm{RSs}$ received by UE and is defined as follows.

$$
\begin{aligned}
& \mathbf{n}_{\mathrm{RS}}(t)= \\
& \int_{\psi_{\mathrm{UE}}} \mathbf{g}_{\mathrm{UE}}\left(\psi_{\mathrm{UE}}\right) \sum_{i}^{N_{\mathrm{RS}}} h_{\mathrm{UR}}^{i}\left(\psi_{\mathrm{UE}}, t\right) \beta^{i} n_{i} \mathrm{~d} \psi_{\mathrm{UE}}
\end{aligned}
$$


where $n_{i}$ is the noise signal generated in the RS.

The $N_{\mathrm{UE}} \times 1$ overall noise vector $\boldsymbol{n}(t)$ is defined as follows.

$$
\boldsymbol{n}(t)=\boldsymbol{n}_{\mathrm{UE}}(\mathrm{t})+\mathbf{n}_{\mathrm{RS}}(t)
$$

where $\boldsymbol{n}_{\mathrm{UE}}(\mathrm{t})$ is the $N_{\mathrm{UE}} \times 1$ noise vector generated at UE.

\subsection{MIMO channel capacity with beam selection}

From the analysis above, channel capacity $C$ of SU-Massive MIMO through the artificial MIMO channel can be calculated as follows.

$$
C=\mathrm{B}_{\mathrm{w}} \sum_{k=1}^{M} \log _{2}\left(1+\frac{\lambda_{k} P_{\mathrm{s}}}{M P_{\mathrm{n} k}}\right)
$$

where $\mathrm{B}_{\mathrm{w}}$ is bandwidth, $\lambda_{k}$ is $k$-th largest eigenvalue of the channel Gram matrix $\mathbf{H}(t) \mathbf{H}^{\mathrm{H}}(t)$, $P_{\mathrm{s}}$ is total transmit power at $\mathrm{BS}$, and $P_{\mathrm{n} k}$ is noise power including the effect of multi-hop relay, which is calculated as follows.

$$
P_{\mathrm{n} k}=\mathrm{E}\left[\left|\boldsymbol{u}_{k}^{\mathrm{H}} \mathbf{n}(t)\right|^{2}\right]
$$

where $\boldsymbol{u}_{\boldsymbol{k}}$ is the eigenvector of the channel Gram matrix corresponding to $\lambda_{k}$.

By taking the beam selection into consideration, $C^{\star}$ is the maximized $C$, and is calculated as follows.

$$
C^{\star}=\underset{\mathbf{A}}{\operatorname{argmax}} C
$$

\subsection{Sequential quasi-optimization procedure}

Regarding the beam selection matrix $\mathbf{A}$, the sequential quasi-optimization procedure drawn in Fig. 3 is widely used to avoid the exhaustive search as in [19][20]. Based on the prepared $\mathbf{g}_{\mathrm{BS}}$, the best beams are selected sequentially to increase channel capacity. The beam selection procedure is as follows.

Firstly, the artificial channel response $h_{\mathrm{URB}}\left(\psi_{\mathrm{UE}}, \psi_{\mathrm{BS}}, t\right)$ is calculated by Eq. (4). The initial beam selection matrix $\mathbf{A}_{0}=\mathbf{0}$ is defined.

Next, channel capacity for each remaining beam candidate is calculated. Among the derived capacities, the one that gives the maximum capacity $C_{M \text {,max }}$ is defined as $\mathbf{A}_{M \text {,max }}$. The beam newly turned on in $\mathbf{A}_{M \text {,max }}$ is selected as $M$-th beam and excluded from beam candidates. Then, $C_{M \text {,max }}$ is compared with $C_{M-1, \text { max }}$. If $C_{M, \text { max }}>C_{M-1, \text { max }}$, the number of selected beams is increased. This trial is repeated until $C_{M, \max }<C_{M-1, \text { max }}$. Finally, the optimal beam selection matrix $\mathbf{A}^{\star}=\mathbf{A}_{M-1, \max }$ is determined.

\section{EXPANSION TO MULTI-HOP}

The proposal of massive analog relay MIMO and the derivation of the optimized channel capacity in single-hop relay is presented in Section 2. However, practical environments and available locations for RSs could be much more complex than the ideal assumptions in Section 2. For example, when shapes of streets are irregular and the RSs are unevenly distributed rather than ideally linearly distributed, there would still exist some areas where the mmWave communications are not possible even if the signals have been relayed for single hop.

In order to address the problem, in this section, the proposed system is extended from a single-hop to multi-hop relay system, in which the artificial MIMO channels are actively generated by multi-hop AF RSs. As shown in Fig. 4, for simplicity of analysis, all RSs are assumed to be deployed at four corners of the tops of the buildings because of the good view fields. As the extension of the RSs in a onedimensional arrangement in Fig. 1, a twodimensional arrangement is shown in Fig. 4, in which signals from the BS are multi-hop relayed by RSs to the UE.

\subsection{Extension of artificial channel response with multi-hop massive analog relay}

In order to explain the derivation of an artificial channel matrix of the multi-hop relay system, all distributed RSs are virtually arranged in a onedimensional configuration as shown in the first column of RSs in Fig. 5, which helps us to consider a system to transmit signals from the BS to the UE through RSs. In the case of multi-hop,

\section{Start}

(i) Set initial beam selection matrix and calculate channel response

(ii) Increment the number of BS selected beams and calculate the channel capacity

(iii) Compare the derived channel capacities

(iv) Get the maximum channel capacity and the beam selection matrix

$$
\text { End }
$$

Fig. 3 - Sequential quasi-optimization procedure for beam selection matrix $\mathbf{A}$ 


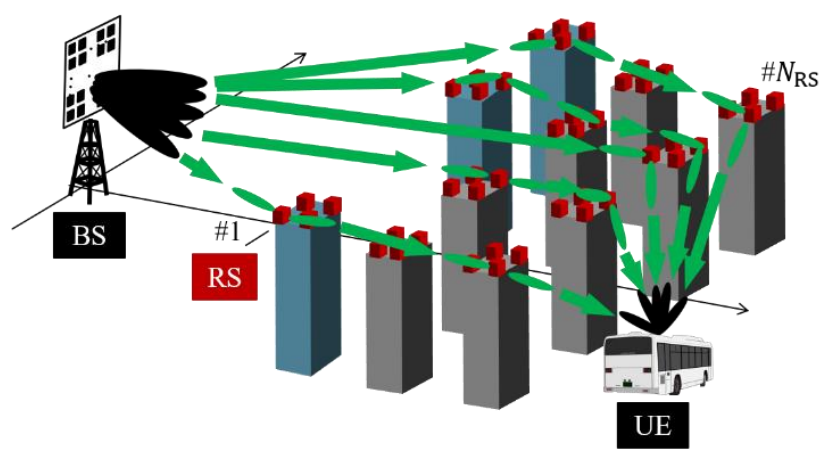

Fig. 4 - System model for mmWave massive multi-hop relay MIMO

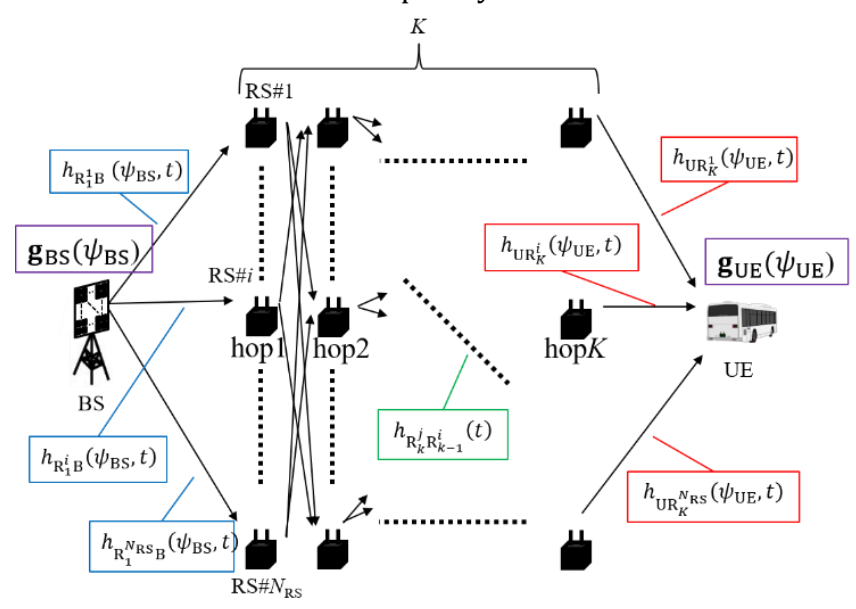

Fig. 5 - Multi-hop artificial channel

this one-dimensional arrangement is repeated for the maximum number of hops. Assuming that the maximum number of hops is $K$, the system consists of $K$ times repeating of the one-dimensional arrangement of RSs as shown in Fig. 5. Finally, it enables us to exhaustively represent all the routes, including routes to the UE with 1 hop, routes with 2 hops, and routes with $K$ hops. Then, RSs and routes for relay are picked up to form the artificial propagation paths.

As shown in Fig. 5, the angles of departure and arrival at the $k$-th hop of the $\mathrm{RS}_{i}$ in the receiving side and transmitting side are defined as $\psi_{\mathrm{R}_{k}^{i} \mathrm{r}}=\left(\theta_{\mathrm{R}_{k}^{i} \mathrm{r}}, \phi_{\mathrm{R}_{k}^{i} \mathrm{r}}\right)$ and $\psi_{\mathrm{R}_{k}^{i} \mathrm{t}}=\left(\theta_{\mathrm{R}_{k}^{i} t}, \phi_{\mathrm{R}_{k}^{i} t}\right)$, where $i=1, \ldots, N_{\mathrm{RS}}$ is the RS index, $k=1, \ldots, K$ is the number of hop, and $K$ is the maximum number of hops. Both receivers and the transmitters of RSs are assumed to be able to actively perform beamforming. The channel response between the BS and $\mathrm{RS}_{i}$, denoted by $h_{\mathrm{R}_{1}^{i} \mathrm{~B}}\left(\psi_{\mathrm{BS}}, t\right)$, is defined as follows.

$$
\begin{aligned}
& h_{\mathrm{R}_{1}^{i} \mathrm{~B}}\left(\psi_{\mathrm{BS}}, t\right)= \\
& \int_{\psi_{\mathrm{R}_{1}^{i} \mathrm{r}}} g_{\mathrm{R}_{1}^{i} \mathrm{r}}\left(\psi_{\mathrm{R}_{1}^{i}}, t\right) h_{\mathrm{R}_{1}^{i} \mathrm{~B}}\left(\psi_{\mathrm{R}_{1}^{i} \mathrm{r}} \psi_{\mathrm{BS}}, t\right) \mathrm{d} \psi_{\mathrm{R}_{1}^{i} \mathrm{r}}
\end{aligned}
$$

where $g_{\mathrm{R}_{1}^{i} \mathrm{r}}$ is the receiving antenna directivity of the 1st-hop $\mathrm{RS}_{i}$.

The channel response between $k-1$ th hop in $\mathrm{RS}_{i}$ and $k$-th hop in $\mathrm{RS}_{j}$, denoted by $h_{\mathrm{R}_{k}^{j}} \mathrm{R}_{k-1}^{i}(t)$, is defined as follows.

$$
\begin{array}{r}
h_{\mathrm{R}_{k}^{j} \mathrm{R}_{k-1}^{i}}(t)=\int_{\psi_{\mathrm{R}_{k}^{j}}{ }^{\mathrm{r}}} \int_{\psi_{\mathrm{R}_{k-1}^{i}} \mathrm{t}} g_{\mathrm{R}_{k}^{j} \mathrm{r}}\left(\psi_{\mathrm{R}_{k}^{j} \mathrm{r}^{1}}, t\right) \\
\cdot h_{\mathrm{R}_{k}^{j} \mathrm{R}_{k-1}^{i}}\left(\psi_{\mathrm{R}_{k}^{j} \mathrm{r}^{\prime}} \psi_{\mathrm{R}_{k-1}^{i} \mathrm{t}^{\prime}} t\right) \\
\cdot g_{\mathrm{R}_{k-1}^{i} \mathrm{t}}\left(\psi_{\mathrm{R}_{k-1}^{i} \mathrm{t}^{\prime}} t\right) \\
\mathrm{d} \psi_{\mathrm{R}_{k-1}^{i}} \mathrm{t} \psi_{\mathrm{R}_{k}^{j} \mathrm{r}}
\end{array}
$$

where $j=1, \ldots, N_{\mathrm{RS}}$ is the RS index in $k$-th hop, $g_{\mathrm{R}_{k-1}^{i} \mathrm{t}}, g_{\mathrm{R}_{k}^{j} \mathrm{r}}$ are the antenna directivities in $k-1$ th hop in $\mathrm{RS}_{i}$ in the transmitting side, and in $k$-th hop in $\mathrm{RS}_{j}$ in the receiving side.

The channel response between the $k$-th hop in $\mathrm{RS}_{i}$ and the UE, denoted by $h_{\mathrm{UR}_{k}^{j}}\left(\psi_{\mathrm{UE}}, t\right)$, is defined as follows.

$$
\begin{array}{r}
h_{\mathrm{UR}_{k}^{j}}\left(\psi_{\mathrm{UE}}, t\right)=\int_{\psi_{\mathrm{R}_{k}^{j} \mathrm{t}}} h_{\mathrm{UR}_{k}^{j}}\left(\psi_{\mathrm{UE}}, \psi_{\mathrm{R}_{k}^{j} \mathrm{t}^{\prime}} t\right) \\
\cdot g_{\mathrm{R}_{k}^{j} \mathrm{t}}\left(\psi_{\mathrm{R}_{k}^{j} \mathrm{t}^{\prime}}, t\right) \mathrm{d} \psi_{\mathrm{R}_{k}^{j} \mathrm{t}}
\end{array}
$$

where $g_{\mathrm{R}_{k}^{j} \mathrm{t}}$ is the antenna directivity in $k$-th hop in the $\mathrm{RS}_{i}$ (transmitting side).

The single-hop channel response between the BS and UE using $\mathrm{RS}_{i}$ at 1st-hop, denoted by $h_{\mathrm{UR}{ }_{1}^{i} \mathrm{~B}}\left(\psi_{\mathrm{UE}}, \psi_{\mathrm{BS}}, t\right)$, is defined as follows.

$$
\begin{aligned}
& h_{\mathrm{UR}_{1}^{i} \mathrm{~B}}\left(\psi_{\mathrm{UE}}, \psi_{\mathrm{BS}}, t\right)= \\
& \quad h_{\mathrm{UR}_{1}^{i}}\left(\psi_{\mathrm{UE}}, t\right) \beta^{i} h_{\mathrm{R}_{1}^{i} \mathrm{~B}}\left(\psi_{\mathrm{BS}}, t\right)
\end{aligned}
$$

The channel response between the BS and UE with $K$ hops, denoted by $h_{\mathrm{UR}_{K}^{p} \mathrm{R}_{K-1}^{q} \cdots \mathrm{R}_{1}^{i} \mathrm{~B}}\left(\psi_{\mathrm{UE}}, \psi_{\mathrm{BS}}, t\right)$, is defined as follows. 


$$
\begin{aligned}
& h_{\mathrm{UR}_{K}^{p} \mathrm{R}_{K-1}^{q} \cdots \mathrm{R}_{1}^{i} \mathrm{~B}}\left(\psi_{\mathrm{UE}}, \psi_{\mathrm{BS}}, t\right) \\
& =h_{\mathrm{UR}_{K}^{p}}\left(\psi_{\mathrm{UE}}, t\right) \beta^{p} h_{\mathrm{R}_{K}^{p} \mathrm{R}_{K-1}^{q}}(t) \\
& \cdot \beta^{q \cdots} \cdots \beta^{i} h_{\mathrm{R}_{1}^{i} \mathrm{~B}}\left(\psi_{\mathrm{BS}}, t\right)
\end{aligned}
$$

where $p, q, \cdots=1, \ldots, N_{\mathrm{RS}}$ is the RS index in $K$-1th hop and $K$ hop. Although $p \neq q \neq \cdots \neq i$ to avoid the self-loops and to avoid multiple use of the same RS in different paths, those constraints are applied by the routing algorithm in Section 3.3.

By summing up the above derivations, the channel response between the BS and UE, with considerations of all paths including self-loops, denoted by $h_{\mathrm{URB}}\left(\psi_{\mathrm{UE}}, \psi_{\mathrm{BS}}, t\right)$, can be defined as follows.

$$
\begin{aligned}
& h_{\mathrm{URB}}\left(\psi_{\mathrm{UE}}, \psi_{\mathrm{BS}}, t\right)=h_{\mathrm{UB}}\left(\psi_{\mathrm{UE}}, \psi_{\mathrm{BS}}, t\right) \\
& +\sum_{i=1}^{N_{\mathrm{RS}}} h_{\mathrm{UR}_{1}^{i} \mathrm{~B}}\left(\psi_{\mathrm{UE}}, \psi_{\mathrm{BS}}, t\right) \\
& +\sum_{j=1}^{N_{\mathrm{RS}}} \sum_{i=1}^{N_{\mathrm{RS}}} h_{\mathrm{UR}_{2}^{j} \mathrm{R}_{1}^{i} \mathrm{~B}}\left(\psi_{\mathrm{UE}}, \psi_{\mathrm{BS}}, t\right)+\cdots \\
& +\sum \cdots \sum_{K} h_{\mathrm{UR}_{K}^{p} \mathrm{R}_{K-1}^{q} \cdots \mathrm{R}_{1}^{i} \mathrm{~B}}\left(\psi_{\mathrm{UE}}, \psi_{\mathrm{BS}}, t\right)
\end{aligned}
$$

where $h_{\mathrm{UB}}\left(\psi_{\mathrm{UE}}, \psi_{\mathrm{BS}}, t\right)$ is the channel response of the direct path between the BS and UE.

\subsection{Noise vector with multi-hop analog relay}

The $N_{\mathrm{UE}} \times 1$ noise vector from 1 st to $K$-th hop RSs $\mathbf{n}_{\mathrm{RS}}(t)$ is defined as follows.

$$
\begin{aligned}
& \mathbf{n}_{\mathrm{RS}}(t) \\
& =\int_{\psi_{\mathrm{UE}}} \mathbf{g}_{\mathrm{UE}}\left(\psi_{\mathrm{UE}}\right) \sum_{i=1}^{N_{\mathrm{RS}}}\left(h_{\mathrm{UR}_{1}^{i}}\left(\psi_{\mathrm{UE}}, t\right)\right. \\
& +\sum_{j=1}^{N_{\mathrm{RS}}} h_{\mathrm{UR}_{2}^{j}}\left(\psi_{\mathrm{UE}}, t\right) \beta^{j} h_{\mathrm{UR}_{2}^{j} \mathrm{R}_{1}^{i} \mathrm{~B}}(t) \\
& +\cdots \sum_{K-1} \ldots \sum_{\mathrm{UR}_{K}^{p}}\left(\psi_{\mathrm{UE}}, t\right) \\
& \left.\left.\cdot \beta^{p} \cdots \beta^{j} h_{\mathrm{UR}_{2}^{j} \mathrm{R}_{1}^{i}}(t)\right)^{n_{i}}\right)^{\mathrm{d} \psi_{\mathrm{UE}}}
\end{aligned}
$$

where $n_{i}$ is the noise signal generated in the $\mathrm{RS}_{i}$. In Eq.(18), the RS in the first column of Fig. 5 is a noise source and is amplified and forwarded in each hop.
3.3 Beam selection algorithm based on routing

In order to maximize channel capacity, the optimized routes and corresponding beams of RSs need to be derived through full-search, which traverses all the combinations of the beam selections in the BS and RSs. Assuming that signals are relayed in a maximum number of $K$ hops and the number of available RSs is $N_{\mathrm{RS}}$, the computational cost of such full-search algorithm $E_{\max }$ is described as follows.

$$
E_{\max }=M \sum_{k=1}^{K}\left(N_{\mathrm{RS}} B_{\mathrm{RS}}\right)^{k} N_{\mathrm{BS}}
$$

where $B_{\mathrm{RS}}$ is the number of candidate beams of RS for both sides .

It is obviously indicated by Eq. (19) that the fullsearch algorithm inevitably results in an enormous and unaffordable computational cost. Since the position of the UE could change time by time, the routes and beams need to be recalculated instantaneously according to the new UE position to guarantee the mmWave communication. Therefore, it is neither practical nor feasible to use the fullsearch method for fast moving UEs such as vehicles.

In order to complete the selection of beams in real time, we propose an algorithm to determine relay routes and corresponding beams of RSs sequentially by finding the shortest paths.

Fig. 6 shows the proposed routing algorithm for the massive multi-hop relay MIMO system. It is assumed that the BS knows all RS locations in advance and can obtain UE location in real time. The BS finds routes based on the proposed algorithm and specifies the relay's beam indices. It is assumed that an RS is used only for a single route since the signal multiplexing capability is not considered for RSs in this paper. Blockage between RSs and the UE by moving objects, such as vehicle, is ignored. First, in Step I, the relative positions and distances among the BS, RSs, and UE are calculated according to the real-time location of UE. It is noted that distances between the BS and RSs are fixed, while the distances between RSs and the UE are varying quickly. Next, as shown in Step II, a graph of artificial channels is built, in which nodes are BS, RSs and UE, and weighted edges connect all nodes. The distance between two nodes is used as a weight, and the edges of self-loops and NLOS links are removed from the graph. Since it is assumed that 


\section{Start}

(i) Determine distances among BS, RSs and UE

(ii) Setting weights and removing unavailable links

(iii) Selecting RSs to relay between BS and UE by the shortest path

(iv) Removing selected route and RSs from the artificial channel

\section{End}

Fig. 6 - Routing algorithm flow chart

the locations of all nodes are ideally collected, NLOS links can be identified by geographical information. Then, in Step III, the route with the shortest path between the BS and UE is calculated by Dijkstra's algorithm [21], instead of traversing the channel capacities of all possible routes. After a route is selected, RSs in the selected route are excluded from the graph in Step IV. And then, the process of finding the next shortest path is started. Path decisions should be repeated until there are no more RSs that can be used for relaying.

After all the paths and corresponding beams have been selected, the number of streams (paths) is optimized using the algorithm shown in Fig. 3. Finally, the channel capacity of massive multi-hop relay MIMO is calculated as in Section 2.

\section{NUMERICAL ANALYSIS}

In this section, a simulation environment is explained, and performance of the proposed mmWave massive analog relay MIMO system is comprehensively evaluated.

\subsection{Simulation environment}

\subsubsection{Simulation model}

Table 1 shows parameters used for the analysis. In the simulation, the buildings are squares with sizes of $20 \mathrm{~m} \times 20 \mathrm{~m}$, and RSs are placed on each building. It is assumed that there are roads between each building, and the interval between each building is $10 \mathrm{~m}$. Regarding the channel model, LOS path and single bounce reflected paths between the BS, RSs, and UE are taken into account.

System performances in two layouts are evaluated: I. The one-dimensional arrangement model shown in Fig. 7. II. The two-dimensional arrangement model shown in Fig. 8. In Case I, RSs are arranged linearly at equal intervals $(30 \mathrm{~m})$, and totally $64 \mathrm{RSs}$ are deployed. In Case II, RSs are deployed at four corners on tops of buildings, and totally 64 RSs are deployed in 16 buildings in a gridded layout. 12 of the 16 buildings are $20 \mathrm{~m}$ height, which are marked as blue buildings in Fig. 8, and the rest are $30 \mathrm{~m}$ height. The UE, which is assumed to be a vehicle, moves between buildings. The heights of the BS and $\mathrm{UE}$ are $35 \mathrm{~m}$ and $3 \mathrm{~m}$, respectively.

\subsubsection{Antenna characteristics}

\section{(a) BS antenna}

Two-dimensionally arranged planar patch antenna elements are used as BS antennas, and hybrid BF is adopted. There are two types of hybrid beamforming: a full array type that uses all antenna elements and a sub-array type that uses some antenna elements. In this paper, we use the full-array type that has higher performance than the sub-array type. Directivity of each patch antenna element follows 3GPP Release 15 [22]. $N_{\mathrm{BS}}=80 \times 80$ beam candidates covering both azimuth and elevation angles are calculated by 2-D Discrete Fourier Transform (DFT) beam patterns with oversampling.

Table 1 - Parameters used in the analysis

\begin{tabular}{|c|c|}
\hline Bandwidth & $400 \mathrm{MHz}$ \\
\hline Carrier frequency & $28 \mathrm{GHz}$ \\
\hline $\begin{array}{l}\text { Number of nodes } \\
\text { (BS/RS/UE) }\end{array}$ & $1 / 64 / 1$ \\
\hline Antenna elements (BS) [17] & $\begin{array}{l}\text { Planar patch } \\
\text { antenna }\end{array}$ \\
\hline $\begin{array}{l}\text { Number of antenna beams } \\
\text { (BS/UE) }\end{array}$ & $72 / 72$ \\
\hline Antenna elements (UE) & Sector antenna \\
\hline Directivity gain at UE & $20 \mathrm{~dB}$ \\
\hline $\begin{array}{c}\text { Height of nodes } \\
\text { (BS/RS(high)/RS(low)/UE) }\end{array}$ & $\begin{array}{c}35 \mathrm{~m} / 30 \mathrm{~m} / 20 \mathrm{~m} \\
/ 3 \mathrm{~m}\end{array}$ \\
\hline Free Space Path Loss (dB) & $20 \log _{10} \frac{4 \pi d}{\lambda}$ \\
\hline RS amplification gain & $25 \mathrm{~dB}$ \\
\hline Directivity gain at $\mathrm{RS}(\mathrm{Rx} / \mathrm{Tx})$ & $30 \mathrm{~dB} / 30 \mathrm{~dB}$ \\
\hline Transmitting power (EIRP) & $25 \mathrm{dBm} / \mathrm{MHz}$ \\
\hline Noise figure & $10 \mathrm{~dB}$ \\
\hline
\end{tabular}


(b) UE antenna

In this paper, $N_{\mathrm{UE}}=72$ antenna beams are modeled by ideal sector antenna covering all upper hemi-sphere [23].

\section{(c) RS antenna}

All RS antennas are assumed to have ideal beam control and always direct to the target antenna with the maximum directivity gain. The sinc function is used as the antenna directivity pattern of RSs [24].

\subsection{Results of numerical analysis}

Based on simulation configurations explained above, system performance of the proposed massive analog relay MIMO for mmWave communications is evaluated by numerical simulations.

\subsubsection{One-dimensional arrangement model}

For the one-dimensional case, Fig. 9 and Fig. 10 show channel capacity and number of streams plotted every $15 \mathrm{~m}$ when the UE moves straight

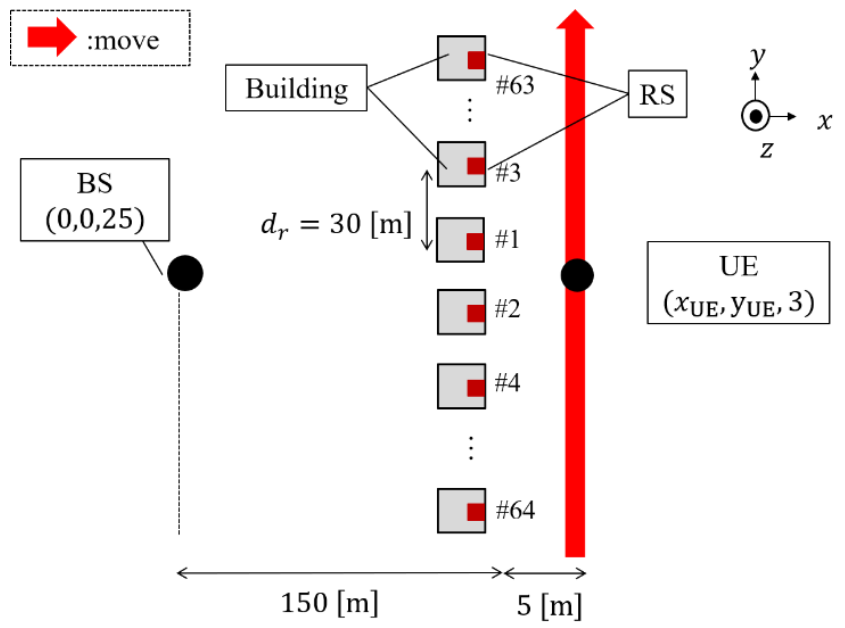

Fig. 7 - One-dimensional arrangement model

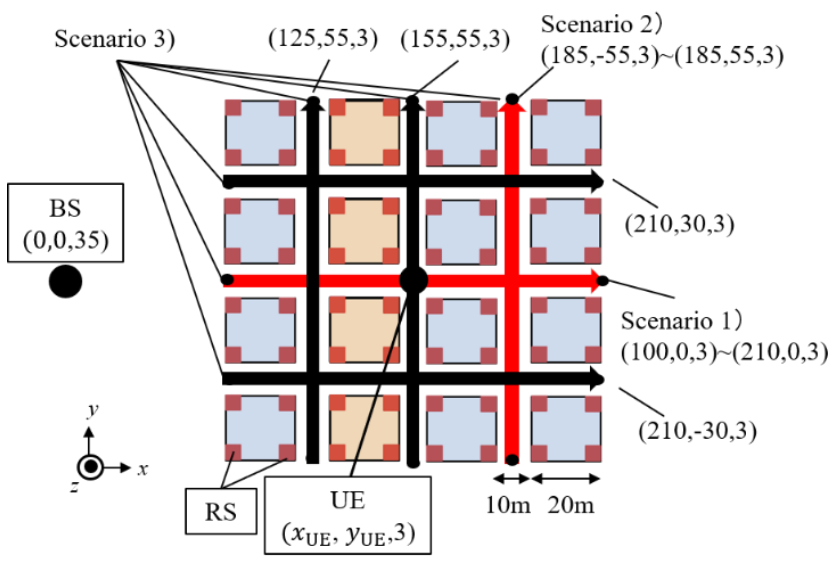

Fig. 8 - Two-dimensional arrangement model from $(155,-960,3)$ to $(155,960,3)$. It can be seen that in the conventional method (w/o RS), in most UE positions, mmWave communications are not possible due to the blockage effects of buildings. In addition, even at positions where the channel capacity is obtained, the number of the streams is only one, indicating that no reflected wave can be obtained and MIMO transmission is not possible. On the other hand, in the proposed method, channel capacities of about 5Gbps or more is obtained regardless of UE position, and number of streams is greatly improved, indicating that MIMO transmission is possible. At positions where channel capacity was able to be obtained by LOS path, i.e., $\mathrm{y}_{\mathrm{UE}}=-30,0,30 \mathrm{~m}$, further increase on channel capacity can be obtained.

Fig. 11 shows dependency of channel capacity on the available number of RSs. To evaluate capacities with a different number of RSs, RSs are turned on in order from \#1 to \#64 as shown in Fig. 7. Evaluations

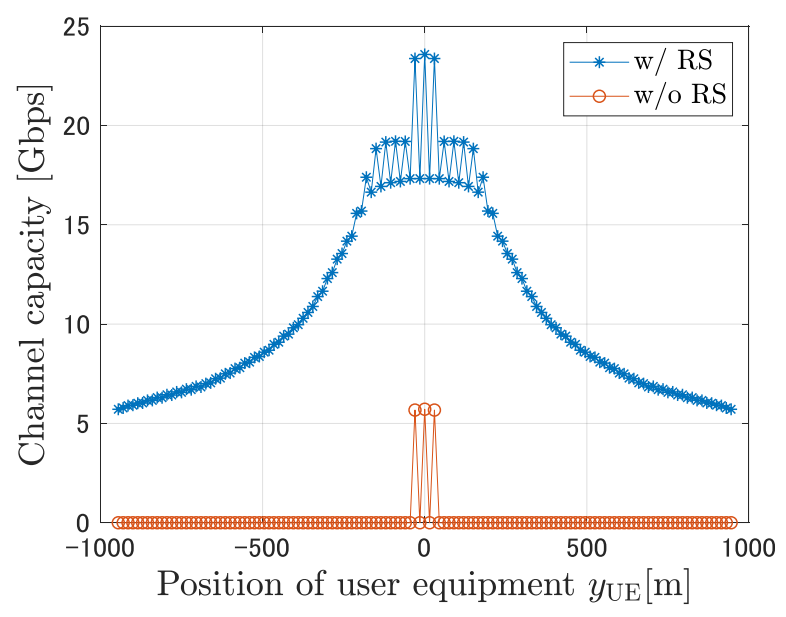

Fig. 9 - Position of UE and channel capacity in one-dimensional arrangement model

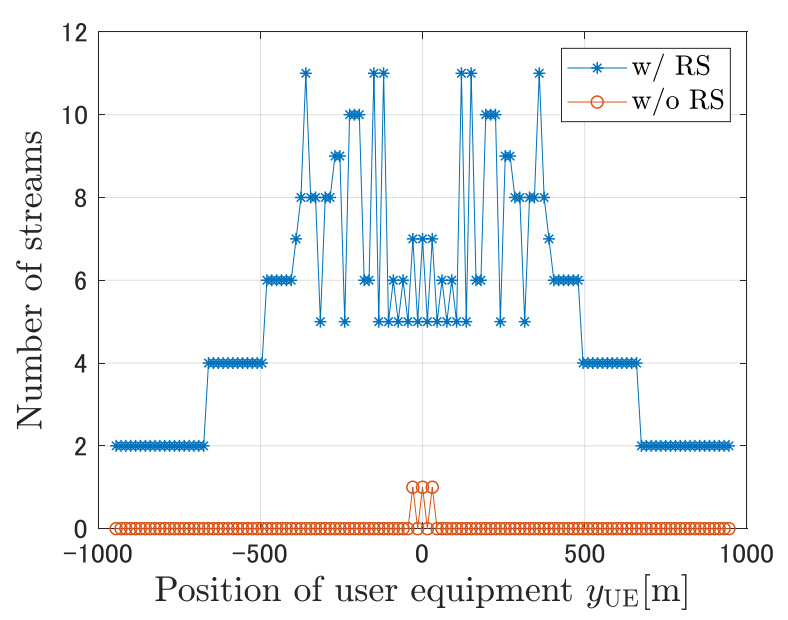

Fig. 10 - Position of UE and number of streams in onedimensional arrangement model 
are done at three different UE positions $y_{U E}=0,150,360 \mathrm{~m}$. It can be seen that as the number of RSs is increased, channel capacity increases, whereas when a certain number of RSs are used, there is no more significant improvement of channel capacity. From different UE positions, it can be understood that significant improvements of channel capacity are contributed by RSs closer to the UE. It indicates the effectiveness of dynamic RS selection to improve channel capacity, and remaining RSs can be turned off to save power consumption.

\subsubsection{Two-dimensional arrangement model}

For the two-dimensional case, system performance of multi-hop massive relay MIMO for mmWave communications is evaluated in three different conditions: Condition 1) LOS environment where the UE moves along $x$-direction from $(100,0,3)$ to $(210,0,3)$ in Fig. 8; Condition 2) NLOS environment where the UE moves along $y$-direction from $(185,-55,3)$ to $(185,55,3)$ in Fig. 8; Conditional 3) Grid environment where the UE moves in a grid among buildings in Fig. 8.

\section{1) LOS environment}

Fig. 12 and Fig. 13 show channel capacities and number of streams plotted every $5 \mathrm{~m}$ when the UE moves along the $x$-axis from $(100,0,3)$ to $(210,0,3)$. Both single-hop and multi-hop relays improve channel capacities when compared with the conventional (w/o hop) method. Channel capacities of more than $20 \mathrm{Gbps}$ and $25 \mathrm{Gbps}$ can be respectively obtained. Multi-hop achieves a further improvement over single-hop, especially at intersections such as at $x_{\mathrm{UE}}=185$ where available RSs for single-hop are blocked by buildings. The

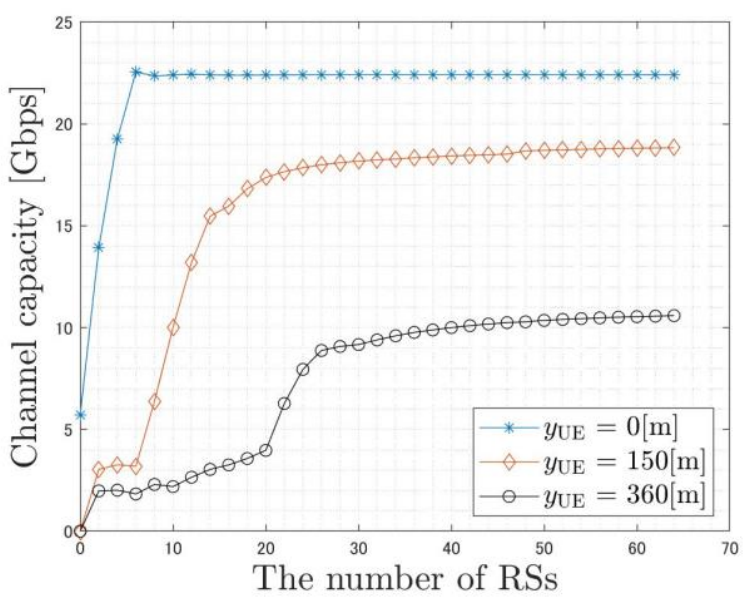

Fig. 11 - The number of RS and channel capacity in one-dimensional arrangement model result in Fig. 13 shows that the number of streams in the LOS environment without hop is only one, which indicates that MIMO transmission is not possible. The number of streams is obtained by both single-hop and multi-hop more than seven, and it enables MIMO communications so that channel capacity is greatly improved. Especially at intersections, number of streams is further increased in the multi-hop case compared to the single-hop, because there are more available RSs around these positions to create artificial channels.

\section{2) NLOS environment}

Fig. 14 and Fig. 15 show channel capacities and number of streams plotted every $5 \mathrm{~m}$ when UE moves along $y$-direction from $(185,-55,3)$ to $(185,55,3)$. In the case of w/o hop, the result clearly shows that without RS, mmWave communications are only possible when LOS path between the BS and UE exists. In the single-hop case, channel capacity is improved in both LOS and NLOS positions compared

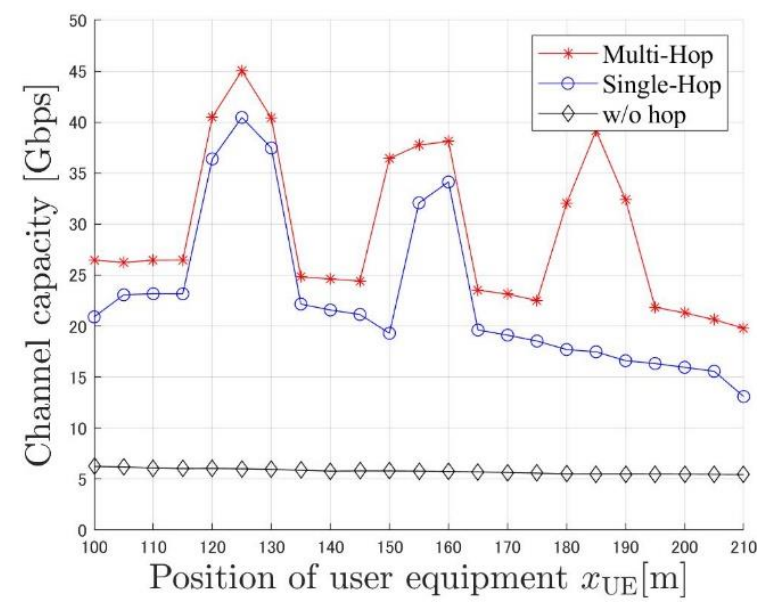

Fig. 12 - Position of UE(x-direction) and channel capacity $\left(y_{U E}=0\right)$

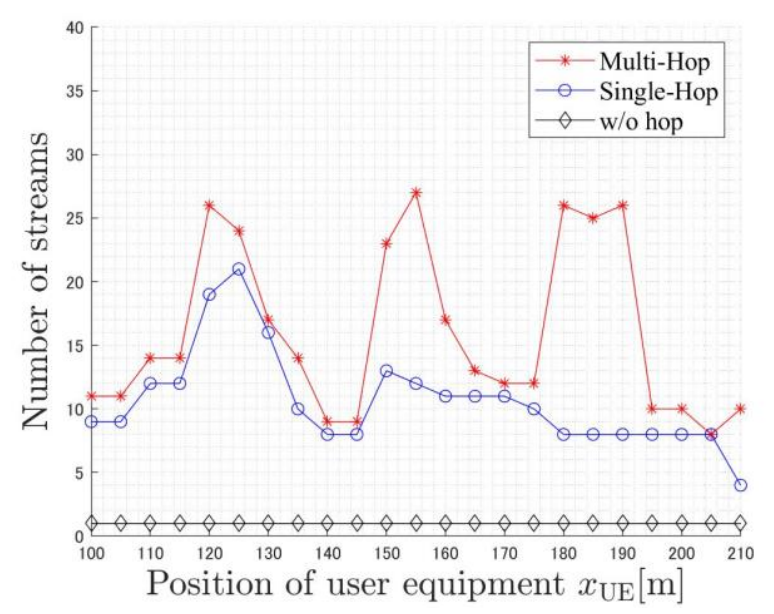

Fig. 13 - Position of UE (x-direction) and number of streams $\left(y_{U E}=0\right)$ 


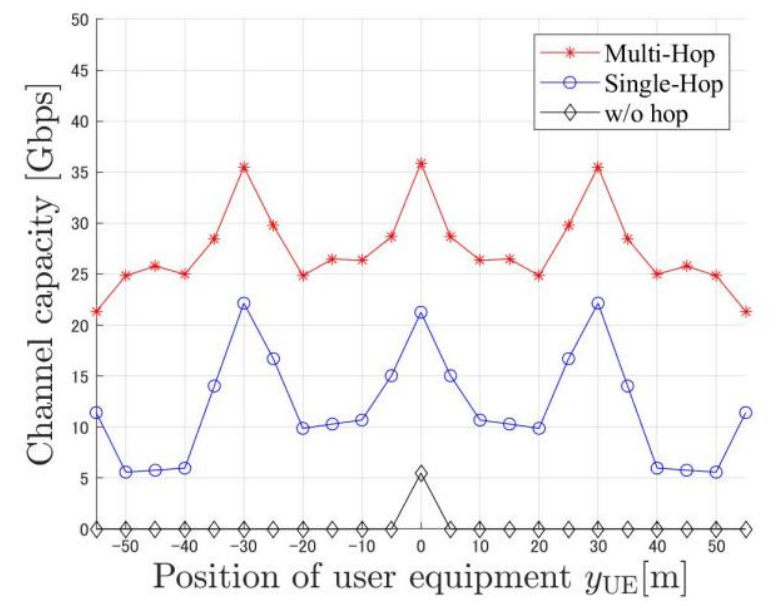

Fig. 14 - Position of UE (y-direction) and channel capacity $\left(x_{U E}=185\right)$

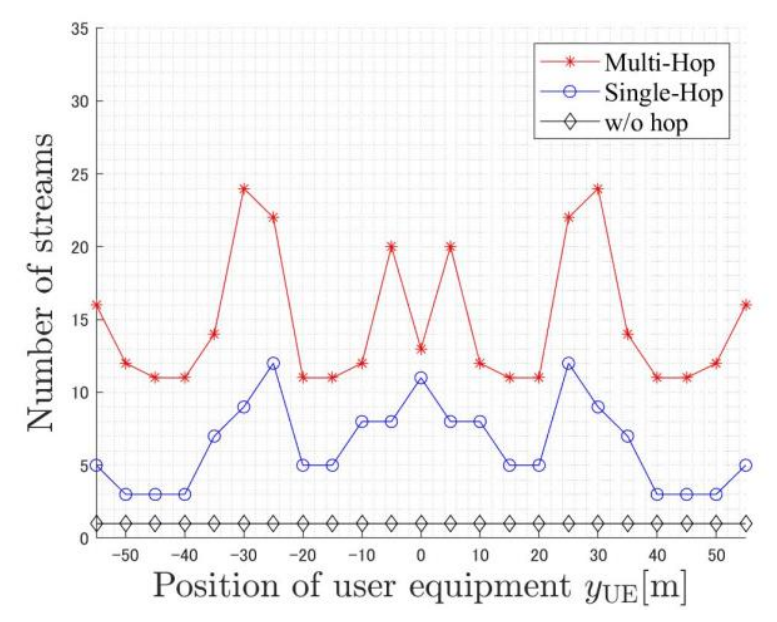

Fig. 15 - Position of UE (y-direction) and number of streams $\left(x_{U E}=185\right)$

with w/o hop. In the multi-hop case by multiple RSs, the proposed system significantly improves channel capacity compared with the single-hop and w/o hop cases. Channel capacities of more than $20 \mathrm{Gbps}$ are archived in all positions. The improvement is particularly obvious at intersections $\left(y_{\mathrm{UE}}=-30,0,30\right)$, where the number of streams is greatly improved by using a large number of RSs deployed along the $x$-direction. Compared with single-hop, the multi-hop case further increases about 15 Gbps channel capacity in all positions. In Fig. 15, in the w/o hop case, in most of the positions, the communication is not possible due to blockage by buildings. On the other hand, the number of streams in single-hop and multi-hop is more than three, so that the MIMO transmission can be performed. In addition, multi-hop relay obtains more streams than single-hop, which leads to further improvement in channel capacity. It can also be seen that the number of streams increases especially at the intersections in NLOS.

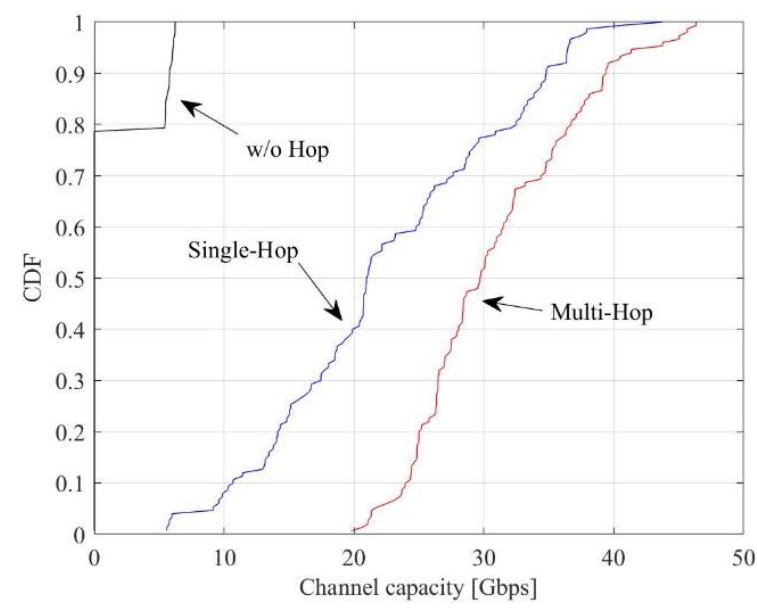

Fig. 16 - CDF of channel capacity multi-hop vs single-hop vs w/o hop

\section{3) Grid environment}

Fig. 16 shows the Cumulative Distribution Function (CDF) of channel capacities for cases of multi-hop, single-hop, and w/o hop. Channel capacity is calculated every $5 \mathrm{~m}$ in a gridded arrangement shown in Fig. 8. Results show that without RSs, channel capacity is 0Gbps, namely mmWave communications are not available in around $80 \%$ of the area in the environment. On the other hand, by hopping with RSs, channel capacity is greatly improved and $100 \%$ coverage is available. Compared with the single-hop case, the multi-hop case can further improve channel capacity of about 12 Gbps with about 10\% cumulative probability. More importantly, the steepness of the curve of the multi-hop case also indicates that it does not only improve channel capacity but also provides stable mmWave communications regardless of locations.

\subsection{Discussions}

In the previous subsections, we have shown the effectiveness of the proposed mmWave massive analog relay MIMO system in a simple scenario with a single BS and single UE. At the last part of this section, generalization of the proposed system to scenarios with multiple UEs and multiple BSs is discussed.

With respect to the multiple UEs, the proposed system can be applied directly in the case of Time Division Multiple Access (TDMA) of UEs or TDMA of groups of UEs. This scenario includes an urban city environment with multiple moving hotspots such as buses where multiple UEs in a hotspot create a group to share the same beams and routes. In addition to the algorithms developed in this paper, further discussions about synchronous beam 
switching among multiple RSs are needed. In the case of Space Division Multiple Access (SDMA), the proposed system can be extended by introducing Multi-User (MU) MIMO pre/post coders. Although the number of RSs per user might be reduced compared with SU-MIMO, the sum rate might be improved if the locations of users separate enough. Furthermore, inter-user interference can be actively reduced by RSs with route and beam optimization.

With respect to the multiple BSs, the proposed system can be extended by introducing coordinated scheduling (UE and RS selection) among multiple BSs. Similarly, with the case of SDMA, inter-user interference attached to the different BSs can be actively reduced by RSs with route and beam optimization. As a result, the outage rate at the cell edge might be improved due to the appropriate selection of UEs and active reduction of inter-user interference.

\section{CONCLUSION}

In this paper, we proposed a massive analog relay MIMO system for mmWave communications to generate an artificial multipath environment for the UE by using a massive number of RSs. By using a large number of RSs, the mmWave MIMO transmission is enabled, and the channel capacity is greatly improved. Besides, the proposed system also makes the mmWave communications in the NLOS environment possible. A practical and feasible routing algorithm for deriving multi-hop routes using a large number of RSs was also proposed. Moreover, a comprehensive system performance analysis was conducted for verification. The results confirm that the proposed multi-hop system can dramatically improve system performance and further improve channel capacity compared to the single-hop and w/o hop systems. Furthermore, it was confirmed that the multi-hop relay system can provide high capacity mmWave communications everywhere in the simulation environment. It is noted that the performance of the proposed system could be further improved because the developed routing algorithm did not consider the effect of spatial correlation between the artificial propagation paths. Although it is expected that the performance of the proposed system will be improved by route selection considering the spatial correlation and path gain, there are concerns about increased control overhead. Therefore, the trade-off between the performance and the overhead needs to be studied in the future.

\section{REFERENCES}

[1] Cisco, "Cisco Annual Internet Report (2018-2023) White Paper”, Mar. 2020.

[2] IMT Vision - "Framework and overall objectives of the future development of IMT for 2020 and beyond", Sep. 2015.

[3] T. Rappaport, R. W. Heath, Jr., R. Daniels, and J. N. Murdock, Millimeter Wave Wireless Communications, Pearson Education, Inc., 2014.

[4] K. Sakaguchi, G.K. Tran, H. Shimodaira, S. Nanba, T. Sakurai, K. Takinami, I. Siaud, E.C. Strinati, A. Capone, I. Karls, R. Arefi, and T. Haustein, "Millimeter-wave Evolution for $5 \mathrm{G}$ Cellular Networks," IEICE Trans. Commun., vol. E98-B, no. 3, pp. 338-402, Mar. 2015.

[5] K. Sakaguchi, R. Fukatsu, T. Yu, E. Fukuda, K. Mahler, R. Heath, T. Fujii, K. Takahashi, A. Khoryaev, S. Nagata, and T. Shimizu, "Towards mmWave V2X in 5G and Beyond to Support Automated Driving," IEICE Trans. Commun., vol. E104-B, no.6, Jun. 2021.

[6] L. Kong, M. K. Khan, F. Wu, G. Chen, and P. Zeng "Millimeter-wave wireless communications for IoT-Cloud supported autonomous vehicles: overview, design, and challenges", IEEE Commun. Mag., vol .55, no. 1, Jan. 2017.

[7] A. Benjebbour, M. Iwabuchi, Y. Kishiyama, W. Guangjian, L. Gu, Y. Cui, and T. Takada, "Outdoor experimental trials of long range mobile communications using $39 \mathrm{GHz}$, IEEE VTC-Spring, Jun. 2018.

[8] M. Shafi, A. F. Molisch, P. J. Smith, T. Haustein, P. Zhu, P. De Silva, F. Tufvesson, A. Benjebbour, and G. Wunder, "5G: A tutorial overview of standards trails challenges deployment and practice", IEEE J. Sel. Areas Commun., vol. 35, no. 6, pp. 1201-1221, Jun. 2017.

[9] S. Sun, T. S. Rappaport, R. W. Heath, A. Nix, and S. Rangan, "MIMO for millimeter-wave wireless communications: beamforming, spatial multiplexing, both?", IEEE Commun. Mag., vol. 52, issue 12, Dec. 2014.

[10] D-W. Yue and H. H. Nguyen, "Multiplexing Gain Analysis of mmWave Massive MIMO Systems With Distributed Antenna Subarrays", IEEE Trans. Veh. Technol., vol. 68, issue 11, Nov. 2019. 
[11] H. Ogawa, G. K. Tran, K. Sakaguchi, and T. Haustein, "Traffic Adaptive Formation of mmWave Meshed Backhaul Networks," IEEE ICC, May 2017.

[12] 3GPP TS 38.174, "NR; Integrated Access and Backhaul (IAB) radio transmission and reception (Release 16)", V16.1.0, Jan. 2021.

[13] J. Du, E. Onaran, D. Chizhik, S. Venkatesan, and R. A. Valenzuela, "Gbps user rates using mmWave relayed backhaul with high-gain antennas", IEEE J. Sel. Areas Commun., vol. 35, no. 6, pp. 1363-1372, Jun. 2017.

[14] S. Gong, X. Lu, D. T. Hoang, D. Niyato, L.Shu, D. I. Kim, and Y. Liang, "Toward Smart Wireless Communications via Intelligent Reflecting Surfaces: A Contemporary Survey," IEEE Commun. Surv. Tutor., vol. 22, no. 4 , pp. 2283-2314, 2020.

[15] G. Yang and M. Xiao, "Performance Analysis of Millimeter-Wave Relaying: Impacts of Beamwidth and Self-Interference", IEEE Trans. Commun., vol. 66, issue 2, Feb. 2018.

[16] H. Abbas and K. Hamdi, "Millimeter Wave Communications over Relay Networks", IEEE WCNC, Apr. 2018.

[17] Y. Sugihara and K. Sakaguchi, "mmWave massive analog relay MIMO for improvement of channel capacity”, IEEE WCNCW, Apr. 2020

[18] F. Sohrabi and W. Yu, "Hybrid Analog and Digital Beamforming for mmWave OFDM Large-Scale Antenna Arrays," IEEE J. on Sel. Areas in Commun., vol. 35, no. 7, pp. 1432-1443, Jul. 2017.

[19] Z. Shen, R. Chen, J. G. Andrews, R. W. Heath, and B. L. Evans, "Low complexity user selection algorithms for multiuser MIMO systems with block diagonalization," IEEE Trans. Signal Process., vol.54, no. 9, pp. 3658-3663, Sep. 2006.

[20] Y. Chang and K. Araki, "Simplified capacitybased user scheduling algorithm for multiuser MIMO systems with block diagonalization," IEICE Trans. Commun., vol. E940B, no. 10, pp. 2837-2846, Oct. 2011.

[21] E. W. Dijikstra, "A note on two problems in connection with graphs", Numerishe Mathmatik, vol. 1, no. 1, pp. 269-271, 1959.
[22] 3GPP TR 38.901, “3rd Generation Partnership Project; Technical Specification Group Radio Access Network; Study on channel model for frequencies from 0.5 to $100 \mathrm{GHz}$ (Release 15)”, V15.0.0, Jun. 2018.

[23] T. Bai and R. W. Heath, Jr., "Coverage and rate analysis for millimeter-wave cellular networks," IEEE Trans. Wireless Commun., vol. 14, no. 2, pp. 1100-1114, Feb. 2015.

[24] Y. Zeng and R. Zhang, "Millimeter wave MIMO with lens antenna array: A new path division multiplexing paradigm," IEEE Trans. Commun., vol. 64, no. 4, pp. 1557-1571, Apr. 2016.

\section{AUTHORS}

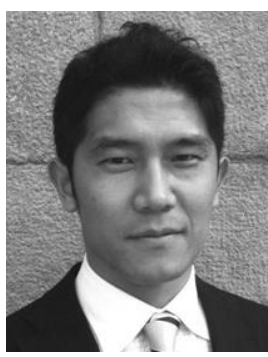

Kei Sakaguchi received an M.E. degree in information processing from Tokyo Institute of Technology in 1998, and a Ph.D degree in electrical \& electronics engineering from Tokyo Institute of Technology in 2006. Currently, he is working at the Tokyo Institute of Technology in Japan as a dean in Tokyo Tech Academy for Super Smart Society and as a professor in the School of Engineering. At the same time, he is working for oRo Co.,Ltd. in Japan as an outside director. He received the Outstanding Paper Awards from SDR Forum and IEICE in 2004 and 2005 respectively, and three Best Paper Awards from IEICE communication society in 2012, 2013, and 2015. He also received the Tutorial Paper Award from IEICE communication society in 2006. His current research interests are in B5G cellular networks, millimeter-wave communications, wireless energy transmission, V2X for automated driving, and super smart society. He is a fellow of IEICE, and a senior member of IEEE.

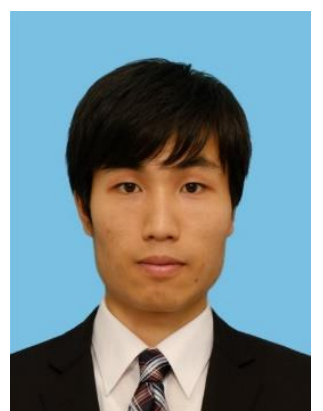

Takumi Yoneda received a B.E. degree in electrical and electronic engineering from Tokyo Institute of Technology in 2020. He is currently pursuing his M.E. degree in the Department of Electrical \& Electronic Engineering, Tokyo Instiitute of Technology. His current research interests are millimeter-wave communications and cellular networks. $\mathrm{He}$ is a student member of IEICE and IEEE. 


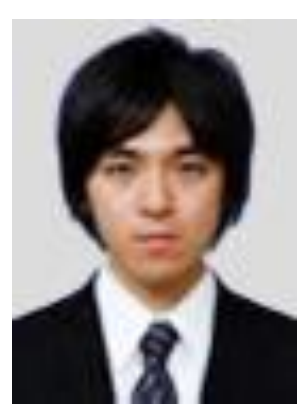

Masashi Iwabuchi received B.S. and M.S. degrees from the Tokyo Institute of Technology, Tokyo, Japan, in 2008 and 2010, respectively. From 2010 to 2016, he joined the NTT Access Network Service Systems Laboratories, Nippon Telegraph and Telephone Corporation (NTT). From 2016 to 2019, he joined NTT DOCOMO, Inc. Since 2019, he has been with NTT again. His current interests are in smart repeaters and reconfigurable intelligent surfaces technologies for $5 \mathrm{G}$ evolution and $6 \mathrm{G}$. He received the Young Engineers Award from the Institute of Electronics, Information and Communication Engineers (IEICE) in 2015. He is a member of the IEICE.

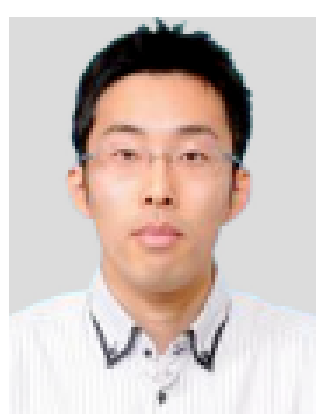

Tomoki Murakami received B.E., M.E., and Dr.Eng. degrees from Waseda University, Japan in 2006, 2008, and 2015. In 2008, he joined Nippon Telegraph and Telephone Corporation (NTT), Yokosuka, Japan. He is currently a research engineer in the Wireless Access Systems Project. His current research interests are high efficiency technologies for future wireless systems. He received the Young Engineer Award from the IEICE in 2010, the Active Research Award from IEICE AP (Antenna and Propagation) in 2010, the Best Tutorial Paper Award from IEICE Communications society in 2014, the Best Paper Award and the KIYASU-Zen'iti Award from the IEICE in 2015, and the Best Paper Award from IEICE on AP in 2016. He is a member of IEEE and IEICE. 